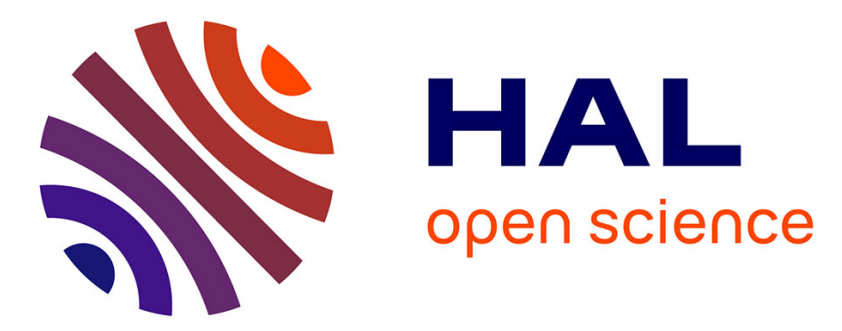

\title{
GENERALIZED OPTICAL BISTABILITY AND CHAOS IN A LASER WITH A SATURABLE ABSORBER
}

Ennio Arimondo, F. de Tomasi, B. Zambon, F. Papoff, Daniel Hennequin

\section{To cite this version:}

Ennio Arimondo, F. de Tomasi, B. Zambon, F. Papoff, Daniel Hennequin. GENERALIZED OPTICAL BISTABILITY AND CHAOS IN A LASER WITH A SATURABLE ABSORBER. Journal de Physique Colloques, 1988, 49 (C2), pp.C2-123-C2-126. 10.1051/jphyscol:1988228 . jpa-00227645

HAL Id: jpa-00227645 https://hal.science/jpa-00227645

Submitted on 1 Jan 1988

HAL is a multi-disciplinary open access archive for the deposit and dissemination of scientific research documents, whether they are published or not. The documents may come from teaching and research institutions in France or abroad, or from public or private research centers.
L'archive ouverte pluridisciplinaire HAL, est destinée au dépôt et à la diffusion de documents scientifiques de niveau recherche, publiés ou non, émanant des établissements d'enseignement et de recherche français ou étrangers, des laboratoires publics ou privés. 
E. ARIMONDO, F. DE TOMASI, B. ZAMBON, F. PAPOFF* and

D. HENNEQUIN* *

Dipartimento di Fisica dell'Universita' di Pisa, Piazza

Torricelli 2, I-56100 Pisa, Italy

* Scuola Normale Superiore, Piazza def Cavalieri, I-56100 Pisa,

Italy

- Laboratoire de spectroscopie Hertzienne, Université de

Lille I, F-59655 Villeneuve-d'Ascq Cedex, France

Resume: Des experiences menees sur un laser a absorbant saturable ont permis de mettre en evidence l'existence de bistabilite' optique generalisee et de chaos dans ce systeme. Des simulations numeriques ont confirme ces resultats.

Abstract .Generalized optical bistability and chaotic behaviour has been observed experimentally and reproduced numerically for a laser with a saturable absorber.

Since the study of the dynamical system theory gained interests among the physics community and the trend of finding prototypes of non-linear systems in the real physical systems started to develop, the laser with a saturable absorber (LSA), although being a system in which strong non-linearities come into play, failed to exhibit the expected behaviour of cascade bifurcations and chaotic dynamics at first experimental and numerical analyses. This indeed seemed rather strange because the " non-linear content " of the ruling equations for this system was quite similar to that of the well known Lorentz set of equations. These equations are given, in the most general form by : (1)

$$
\begin{aligned}
& \dot{\mathrm{I}}=\mathrm{I} *(\mathrm{~A} * \delta-\overline{\mathrm{A}} * \bar{\delta}-1) \\
& \dot{\delta}=-\mathrm{a} * \mathrm{I} * \delta-\int_{0}^{\infty} \mathrm{K}\left(\mathrm{t}^{\prime}\right) *\left(\delta\left(\mathrm{t}-\mathrm{t}^{\prime}\right)-1\right) \\
& \dot{\bar{\delta}}=-\overline{\mathrm{a}} * \mathrm{I} * \bar{\delta}-\int_{0}^{\infty} \overline{\mathrm{K}}\left(t^{\prime}\right) *\left(\delta\left(t-t^{\prime}\right)-1\right)
\end{aligned}
$$

where all the barred quantities refer to the absorber and $K$ and $\bar{K}$ represent the memory functions containing the dynamics of the relaxation including levels different from the resonant ones. However only recently it has been shown that for a range of parameters corresponding to the experimental ones the LSA exhibits a wide variety of dynamical regimes and chaotic behaviours when the analyses with oversimplified models are abandoned and a detailed representation of the memory function is given. The experimental dynamical scenario becomes even more complex and regions of coexistence of two different 
regimes are observed which so far have been only partially reproduced by numerical experiments. In the above set of equations as well in the experimental situation the control parameters have been taken to be $A$ and $\bar{A}$. The first one is proportional to the pumping rate in the gain-medium and the second can be changed by varying the absorber's pressure. A third control parameter, very easy to handle from the experimental point of view, can be chosen to be the cavity detuning, this has the effect of reducing by the same factor $A$ and $a$, as well $\bar{A}$ and $\bar{a}$ in Eqs.(1). If we consider the dependence of the steady state solution from the control parameter $A$ it is found that above a given threshold value $A_{0}$ there are always two solutions ; a trivial one corresponding at $I=0$ which is always unstable and a solution different from zero which can be stable or unstable. The points representative of these solutions in the phase space $I, \delta, \bar{\delta}$ will be denoted by $\mathrm{I}_{\mathrm{O}}$ and $\mathrm{I}_{+}$respectively. The important fact is that the dynamical behaviour of this system can be understood in terms of the local dynamics of the points $I_{0}$ and $I_{+}(2)$. We will consider here the most interesting case in which both points are unstable, which correspond to a range of the parameter $A$ between $A_{0}$ and $A_{H}$. In this case because of lacking of any stationary solution the system must wander in the phase space until it is attracted by a stable orbit. We have identified two types of stable orbits: the first type corresponds to an intensity pulsating solution getting very close to the plane $I=0$ which we will call type-I solution, whereas the second one corresponds to a self-oscillating solution emerging from the Hopf bifurcation occuring at $A=A_{H}$ that we will call type-II solution. Very near to the threshold $A_{0}$ the typeI solution corresponds to an orbit visiting a large portion of the phase space not being influenced by the local dynamics of the point $I_{+}$, we can think at this orbits as originating from an homoclinic orbit of the point $\mathrm{I}_{0}$, (Fig. 1.a). However as we move near $A_{H}$ the previous orbit transforms into one approaching $I_{+}$along the contracting manifold and spiralling outward on the unstable manifold (Fig.1.b). This orbit returns on the plane $I=0$ toward which it is strongly attracted. We could not observe an orbit of this kind not approaching the plane $I=0$, this would be somewhat connected with the existence of Shilnikov chaos in the LSA: since the motion near the plane $I=0$ is essentially bidimensional the homoclinic reinjection mechanism necessary for the occurence of this type of chaos loses its efficiency.
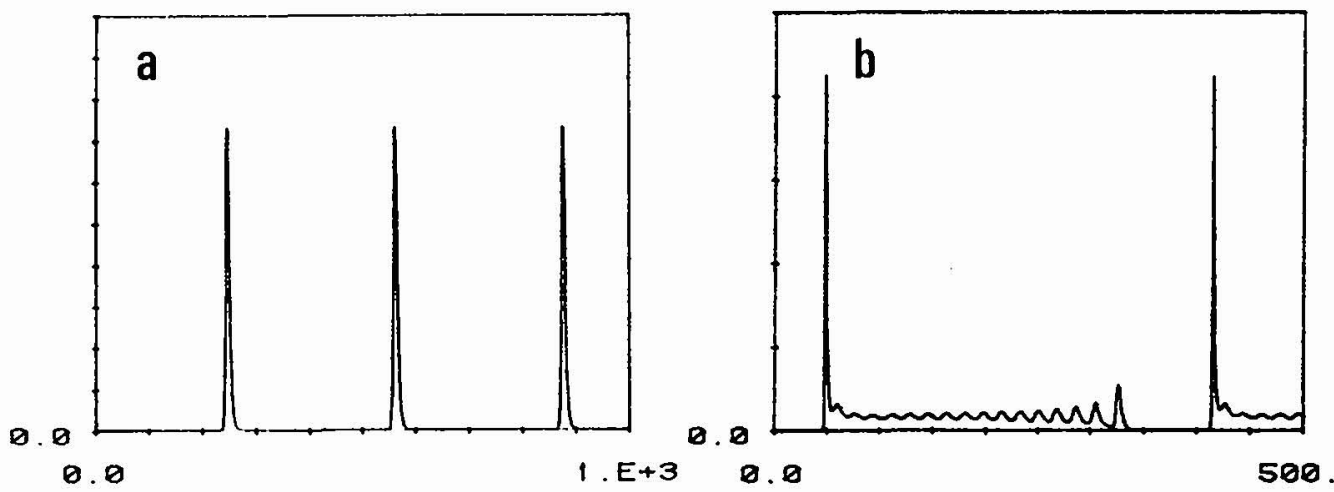

Fig.1. Numerically simulated $P(0)$ type-I pulses (a) and $P(n)$ type-I pulses (b). 
In Fig.(2) we can observe the domain of existence of different regimes of the LSA: Fig.2.a is an experimental phase diagram whereas Fig.2.b is a theoretical one. The type-I solutions are denoted by $P(n)$ with $n$ being the number of spikes of the undulated tail whereas the type-II solutions are denoted by $T$ or $n T$ according to which step of the period doubling sequence they represent. We note that the passage from the pulse $P(n)$ to the pulse $P(n+1)$ occurs discontinuosly and for some values of the control parameters they are separated by a whole period doubling sequence leading to chaotic behaviour. Two types of chaos, one associated with the type-I heteroclinic cycle joining $\mathrm{I}_{0}$ and $\mathrm{I}_{+}$and the other originating from type-II instability are observed on LSA(3).

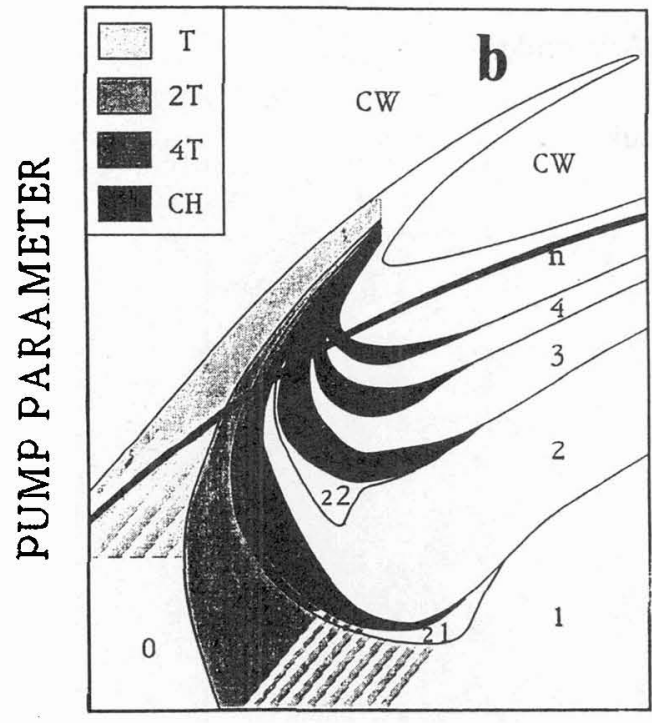

ABSORPTION PARAMETER

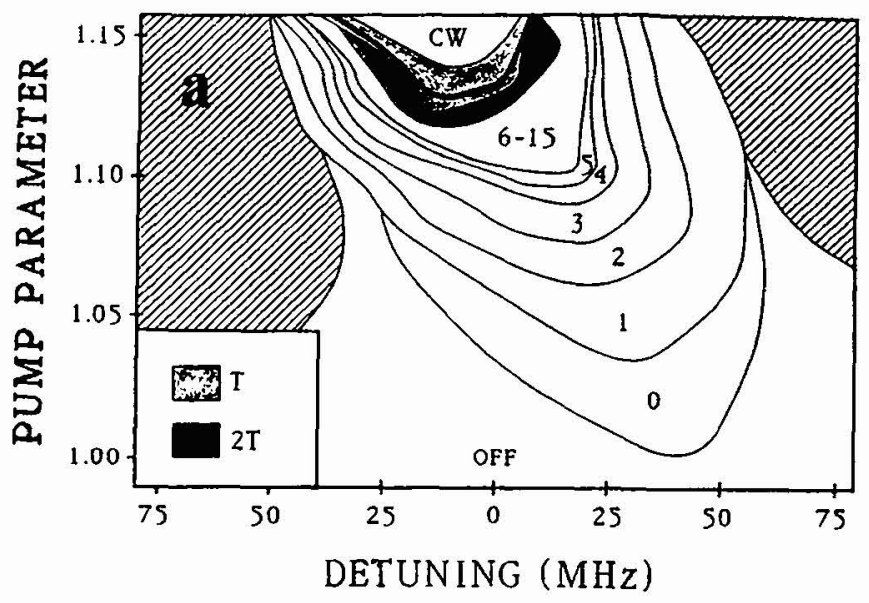

Fig.2. Experimental phase diagram (a). The numbers inside white regions stand for $P(n)$ type-I pulses. The hatched area corresponds to laser-off or to multimode regime. The thick line shows bistability between $P(n)$ and type-II pulses. Phase diagram as derived from numerical simulations (b), the thick line shows bistability between cw and several LSA regimes.

It is well known that LSA presents bistability between the $I_{0}$ and $I_{+}$solutions, however the existence of the type-I and type-II LSA regimes described above leads to the presence of generalized bistability between the different solutions. Bistabilities between type-I instability and $\mathrm{cw}$ regime may appear, as observed on the operation with the $10 \mathrm{P}(20)$ line and low pressure SF6 absorber. For the $10 \mathrm{P}(16)$ line at small frequency detuning a bistability between type-II instability and off regime has been observed. These observations point out that in the LSA phase space, an $I_{+}$stable point and a stable type-I orbit coexists, as well an $I_{0}$ stable point and a stable type-II orbit. On the contrary a bistability between Io and type-I instability is unlikely to occur because type-I orbits evolve in the 
phase space very close to the Io point, that, if stable would attract and destabilize the orbit. To complete the bistability list, a bistability is expected between an $I_{+}$ stable point and a type-II orbit, i.e. the coexistence of a limit cycle around the I+ point independent of the stable $I_{+}$point itself. We have not obtained a clear evidence of this bistable regime in our experimental observations. A last bistability case is between type-I and type-II instabilities, and this case has been illustrated in Fig.2.b. Such a generalized bistability has been observed for type-1 $P(n)$ instabilities at large numbers $n$. Instead a soft continuous transition is observed between type-II and low $n$ type-I instabilities.

References.

(1) B.Zambon, F.De. Tomasi, D.Hennequin and E.Arimondo, to be published

(2) D.Hennequin, F.De. Tomasi, B.Zambon and E.Arimondo

Phys. Rev. A , March 1988

(3) A.Bekkali, F.Papoff, D.Dangoisse, and P.Glorieux

Proceedings of this Conference 